\title{
BIHARMONIC SUBMANIFOLDS IN NONFLAT LORENTZ 3-SPACE FORMS
}

\author{
TORU SASAHARA
}

(Received 10 June 2011)

\begin{abstract}
The purpose of this paper is to classify nonharmonic biharmonic curves and surfaces in de Sitter 3-space and anti-de Sitter 3-space.
\end{abstract}

2010 Mathematics subject classification: primary 53C42; secondary 53B25.

Keywords and phrases: biharmonic curves, biharmonic surfaces, de Sitter space, anti-de Sitter space.

\section{Introduction}

In [8], Eells and Sampson defined biharmonic maps between Riemannian manifolds as an extension of harmonic maps, and Jiang [10] obtained their first and second variational formulas. Since harmonic maps are always biharmonic, it is natural and interesting to investigate nonharmonic biharmonic maps, which are called proper biharmonic maps. A submanifold is called a biharmonic submanifold if the isometric immersion that defines the submanifold is a biharmonic map. During this last decade, many interesting results on proper biharmonic maps and submanifolds have been obtained (see, for example, [3]). In particular, proper biharmonic curves and surfaces in real 3-space forms have been classified (see [4, 5]).

In [12], the author introduced the notion of biharmonic maps between pseudoRiemannian manifolds. If the ambient space is the pseudo-Euclidean space, the notion of biharmonic submanifolds introduced in [12] coincides with Chen's notion of biharmonic submanifolds, that is, submanifolds with harmonic mean curvature vector field (see [6]). Proper biharmonic curves and surfaces in the pseudo-Euclidean 3-space have been classified (see $[6,7]$ ). In particular, it was proved that there exists no proper biharmonic surface in pseudo-Euclidean 3-space.

In this paper, we classify proper biharmonic curves and surfaces in de Sitter 3-space and anti-de Sitter 3-space (Theorems 4.4, 4.5 and 5.4). Contrary to the case of pseudoEuclidean 3-space, there exist proper biharmonic surfaces in those spaces. A further important point is that an example of a proper biharmonic surface in de Sitter space having no Riemannian counterpart is obtained.

(C) 2011 Australian Mathematical Publishing Association Inc. 0004-9727/2011 \$16.00 


\section{Preliminaries}

Let $E_{s}^{n}$ be pseudo-Euclidean $n$-space with metric given by

$$
g=-\sum_{i=1}^{s} d x_{i}^{2}+\sum_{j=s+1}^{n} d x_{j}^{2},
$$

where $\left\{x_{1}, \ldots, x_{n}\right\}$ is the natural coordinate system of $E_{s}^{n}$. Then $E_{s}^{n}$ is a flat pseudoRiemannian manifold with index $s$. We put

$$
\begin{aligned}
& S_{s}^{n}(c)=\left\{\left(x_{1}, \ldots, x_{n+1}\right) \in E_{s}^{n+1} \mid-\sum_{i=1}^{s} x_{i}^{2}+\sum_{j=s+1}^{n+1} x_{j}^{2}=\frac{1}{c}\right\}, \\
& H_{s}^{n}(c)=\left\{\left(x_{1}, \ldots, x_{n+1}\right) \in E_{s+1}^{n+1} \mid-\sum_{i=1}^{s+1} x_{i}^{2}+\sum_{j=s+2}^{n+1} x_{j}^{2}=\frac{1}{c}<0\right\} .
\end{aligned}
$$

These spaces are complete pseudo-Riemannian manifolds with index $s$ of constant curvature $c$. The pseudo-Riemannian manifolds $E_{1}^{n}, S_{1}^{n}(c)$ and $H_{1}^{n}(c)$ are called Minkowski space, de Sitter space and anti-de Sitter space, respectively. These spaces with index 1 are called Lorentz space forms.

Denote $n$-dimensional Lorentz space forms of constant curvature $c$ by $M_{1}^{n}(c)$. The curvature tensor $\tilde{R}$ of $M_{1}^{n}(c)$ is given by

$$
\tilde{R}(X, Y) Z=c(\langle Y, Z\rangle X-\langle X, Z\rangle Y),
$$

where $\langle$,$\rangle is the metric tensor of M_{1}^{n}(c)$.

Let $M^{2}$ be a pseudo-Riemannian surface in $M_{1}^{3}(c)$. We denote by $\nabla$ and $\tilde{\nabla}$ the LeviCivita connections on $M^{2}$ and $M_{1}^{3}(c)$, respectively. Let $X$ and $Y$ be vector fields tangent to $M^{2}$ and let $\xi$ be a normal vector field. Then the formulas of Gauss and Weingarten are given by

$$
\begin{aligned}
\tilde{\nabla}_{X} Y & =\nabla_{X} Y+h(X, Y), \\
\tilde{\nabla}_{X} \xi & =-A_{\xi} X+D_{X} \xi,
\end{aligned}
$$

respectively, where $h, A$ and $D$ are the second fundamental form, the shape operator and the normal connection, respectively. The mean curvature vector field $H$ is defined by $H=\frac{1}{2}$ trace $h$.

Denote by $R$ the curvature tensor of $M^{2}$. Then the equations of Gauss and Codazzi are given respectively by

$$
\begin{aligned}
\langle R(X, Y) Z, W\rangle= & c(\langle X, W\rangle\langle Y, Z\rangle-\langle X, Z\rangle\langle Y, W\rangle) \\
& +\langle h(X, W), h(Y, Z)\rangle-\langle h(X, Z), h(Y, W)\rangle, \\
& \left(\bar{\nabla}_{X} h\right)(Y, Z)=\left(\bar{\nabla}_{Y} h\right)(X, Z),
\end{aligned}
$$


where $X, Y, Z, W$ are vectors tangent to $M$, and $\bar{\nabla} h$ is defined by

$$
\left(\bar{\nabla}_{X} h\right)(Y, Z)=D_{X} h(Y, Z)-h\left(\nabla_{X} Y, Z\right)-h\left(Y, \nabla_{X} Z\right) .
$$

The Laplace operator which acts on the sections of the normal bundle $T^{\perp} M^{2}$ is defined by $\Delta^{D}=-\sum_{i=1}^{2}\left\langle e_{i}, e_{i}\right\rangle\left(D_{e_{i}} D_{e_{i}}-D_{\nabla_{e_{i}} e_{i}}\right)$, where $\left\{e_{i}\right\}$ is a local orthonormal frame of $M^{2}$. The gradient of a function $f$ on $M^{2}$ is defined by $\operatorname{grad} f=-\sum_{i=1}^{2}\left\langle e_{i}, e_{i}\right\rangle\left(e_{i} f\right) e_{i}$.

\section{Biharmonic maps}

Let $M^{m}$ and $N^{n}$ be pseudo-Riemannian manifolds of dimensions $m$ and $n$, respectively, and $\phi: M^{m} \rightarrow N^{n}$ a smooth map. We denote by $\nabla$ and $\tilde{\nabla}$ the Levi-Civita connections on $M^{m}$ and $N^{n}$, respectively. Then the tension field $\tau_{1}(\phi)$ is a section of the vector bundle $\phi^{*} T N^{n}$ defined by

$$
\tau_{1}(\phi):=\operatorname{trace}\left(\nabla^{\phi} d \phi\right)=\sum_{i=1}^{m}\left\langle e_{i}, e_{i}\right\rangle\left(\nabla_{e_{i}}^{\phi} d \phi\left(e_{i}\right)-d \phi\left(\nabla_{e_{i}} e_{i}\right)\right) .
$$

Here $\nabla^{\phi}$ and $\left\{e_{i}\right\}$ denote the induced connection by $\phi$ on the bundle $\phi^{*} T N^{n}$, which is the pull-back of $\tilde{\nabla}$, and a local orthonormal frame field of $M^{m}$, respectively.

A smooth map $\phi$ is called a harmonic map if its tension field vanishes. A map $\phi$ is harmonic if and only if it is a critical point of the energy

$$
E(\phi)=\int \sum_{i=1}^{m}\left\langle d \phi\left(e_{i}\right), d \phi\left(e_{i}\right)\right\rangle d v
$$

under compactly supported infinitesimal variations, where $d v$ is the volume form of $M^{m}$.

We define the bitension field as follows:

$$
\tau_{2}(\phi):=\sum_{i=1}^{m}\left\langle e_{i}, e_{i}\right\rangle\left(\left(\nabla_{e_{i}}^{\phi} \nabla_{e_{i}}^{\phi}-\nabla_{\nabla_{e_{i}} e_{i}}^{\phi}\right) \tau_{1}(\phi)+R^{N}\left(\tau_{1}(\phi), d \phi\left(e_{i}\right)\right) d \phi\left(e_{i}\right)\right),
$$

where $R^{N}$ is the curvature tensor of $N^{n}$. We say that a smooth map $\phi$ is a biharmonic map (or 2-harmonic map) if its bitension field vanishes (see [10,12, 13]). If $\phi$ is a biharmonic isometric immersion, then $M^{m}$ is called a biharmonic submanifold in $N^{n}$. Harmonic maps are clearly biharmonic. Nonharmonic biharmonic maps are called proper biharmonic maps. A biharmonic map $\phi$ is characterised as a critical point of the bienergy

$$
E_{2}(\phi)=\int\left\langle\tau_{1}(\phi), \tau_{1}(\phi)\right\rangle d v
$$

under compactly supported infinitesimal variations (see [10]). For recent information on biharmonic maps, we refer to $[3,11]$.

In the case in which $N^{n}$ is pseudo-Euclidean space and $\phi=\left(\phi_{1}, \ldots, \phi_{n}\right)$ is an isometric immersion, then

$$
\tau_{2}(\phi)=\Delta_{M} \Delta_{M}\left(\phi_{1}, \ldots, \phi_{n}\right)
$$


where $\Delta_{M}=-\sum_{i=1}^{m}\left\langle e_{i}, e_{i}\right\rangle\left(e_{i} e_{i}-\left(\nabla_{e_{i}} e_{i}\right)\right)$. Thus, biharmonicity for an isometric immersion into pseudo-Euclidean space is equivalent to biharmonicity in the sense of Chen (see [6]).

As for proper biharmonic submanifolds in real or Lorentz 3-space forms, the following classification results have been obtained.

THeOREM 3.1 [5]. Let $M$ be a submanifold of real 3-space forms of nonpositive constant sectional curvature. Then $M$ is biharmonic if and only if it is harmonic.

Theorem 3.2 [4]. Let $\gamma: I \rightarrow S^{3}(1)$ be a proper biharmonic unit speed curve and let $x=\mathbf{i} \circ \phi$, where $\mathbf{i}: S^{3}(1) \rightarrow E^{4}$ is the canonical inclusion. Then $x$ is congruent to one of the following two families.

(1) $x(t)=\left(\cos \sqrt{2} t / \sqrt{2}, \sin \sqrt{2} t / \sqrt{2}, d_{1}, d_{2}\right)$, where $d_{1}^{2}+d_{2}^{2}=1$.

$$
x(t)=\left(\frac{\cos \sqrt{1+k} t}{\sqrt{2}}, \frac{\sin \sqrt{1+k} t}{\sqrt{2}}, \frac{\cos \sqrt{1-k} t}{\sqrt{2}}, \frac{\sin \sqrt{1-k} t}{\sqrt{2}}\right),
$$

where $0<k<1$.

TheOREM 3.3 [4]. Let $M^{2}$ be a proper biharmonic surface in $S^{3}(1)$. Then $M^{2}$ is locally a piece of $S^{2}(2) \subset S^{3}(1)$.

Proposition $3.4[6,7]$. Let $x$ be a unit speed curve in $E_{1}^{3}$. Then $x$ is proper biharmonic if and only if $x$ is congruent to one of the following:

(1) a spacelike curve such that $\left\langle x^{\prime \prime}, x^{\prime \prime}\right\rangle=0$, which is given by

$$
x(s)=\left(a s^{3}+b s^{2}, a s^{3}+b s^{2}, s\right)
$$

for some constants $a$ and $b$ satisfying $a^{2}+b^{2} \neq 0$;

(2) a spacelike helix with a spacelike principal normal vector field satisfying $\kappa^{2}=$ $\tau^{2}=a^{2}$

$$
x(s)=\left(\frac{a^{2}}{6} s^{3}, \frac{a}{2} s^{2},-\frac{a^{2}}{6} s^{3}+s\right)
$$

for some nonzero constant $a$;

(3) a timelike helix satisfying $\kappa^{2}=\tau^{2}=a^{2}$;

$$
x(s)=\left(\frac{a^{2}}{6} s^{3}+s, \frac{a^{2}}{6} s^{3}, \frac{a}{2} s^{2}\right)
$$

for some nonzero constant $a$.

Theorem 3.5 [6]. Let $x: M^{2} \rightarrow E_{1}^{3}$ be a biharmonic isometric immersion of a pseudoRiemannian surface $M^{2}$ into $E_{1}^{3}$. Then $x$ is harmonic.

The purpose of this paper is to classify proper biharmonic submanifolds in de Sitter 3-space and anti-de Sitter 3-space. 


\section{Biharmonic curves in nonflat Lorentz 3-space forms}

Let $\left(M_{1}^{3}, g, \tilde{\nabla}\right)$ be a Lorentz 3-manifold and let $\gamma: I \rightarrow M_{1}^{3}$ be a unit speed curve, that is, a curve satisfying $g\left(\gamma^{\prime}, \gamma^{\prime}\right)= \pm 1$. A unit speed curve $\gamma$ is called spacelike (respectively, timelike) if $g\left(\gamma^{\prime}, \gamma^{\prime}\right)=1$ (respectively, $g\left(\gamma^{\prime}, \gamma^{\prime}\right)=-1$ ). A unit speed curve $\gamma$ is said to be a Frenet curve if $g\left(\tilde{\nabla}_{\gamma^{\prime}} \gamma^{\prime}, \tilde{\nabla}_{\gamma^{\prime}} \gamma^{\prime}\right) \neq 0$. A unit speed curve is said to be a geodesic if $\tilde{\nabla}_{\gamma^{\prime}} \gamma^{\prime}=0$.

Let $P=\left(\mathbf{p}_{1}, \mathbf{p}_{2}, \mathbf{p}_{3}\right)$ be an orthonormal frame field along a Frenet curve $\gamma$ such that $\mathbf{p}_{1}=\gamma^{\prime}$ and $P$ satisfies the following Frenet-Serret equations (see [9]):

$$
\tilde{\nabla}_{\gamma^{\prime}} P=P\left(\begin{array}{ccc}
0 & -\epsilon_{1} \kappa & 0 \\
\epsilon_{2} \kappa & 0 & \epsilon_{2} \tau \\
0 & -\epsilon_{3} \tau & 0
\end{array}\right),
$$

where $\kappa$ and $\tau$ are called the curvature and torsion of $\gamma$, respectively, and $\epsilon_{i}=g\left(\mathbf{p}_{i}, \mathbf{p}_{i}\right)$. Note that $\epsilon_{1} \epsilon_{2} \epsilon_{3}=-1$. A unit speed curve is a geodesic if and only if $\kappa=0$ at any point.

The vectors $\mathbf{p}_{2}$ and $\mathbf{p}_{3}$ are called the principal normal vector field and the binormal vector field of $\gamma$, respectively. A Frenet curve with constant curvature and torsion is called a helix. In particular, a helix with zero torsion is called a circle. The mean curvature vector field $H_{\gamma}$ of $\gamma$ is given by $H_{\gamma}=-\epsilon_{3} \kappa \mathbf{p}_{2}$. We remark that $H_{\gamma}=\tau_{1}(\gamma)$.

Let $\gamma: I \rightarrow M_{1}^{3}(c)$ be a Frenet curve. Then, using (2.1) and (3.1), we see that $\gamma$ is biharmonic if and only if the mean curvature vector $H_{\gamma}$ satisfies $\tilde{\Delta} H_{\gamma}=c H_{\gamma}$, where $\tilde{\Delta}$ is the Laplace operator acting on the sections of $\gamma^{*} T M_{1}^{3}(c)$. Applying [9, Theorem 3.2], we have the following proposition.

Proposition 4.1. Let $\gamma: I \rightarrow M_{1}^{3}(c)$ be a Frenet curve. Then $\gamma$ is proper biharmonic if and only if $\gamma$ is a helix with

$$
c=-\epsilon_{3}\left(\epsilon_{1} \kappa^{2}+\epsilon_{3} \tau^{2}\right), \quad \kappa \neq 0 .
$$

Let $E_{t}^{n+1}$ be the corresponding pseudo-Euclidean space where $M_{1}^{n}(c)$ is lying. By a similar computation to [5, Proof of Proposition 4.1], we obtain the following proposition.

Proposition 4.2. Let $\phi: M^{m} \rightarrow M_{1}^{n}(c)$ be an isometric immersion and let $x=\mathbf{i} \circ \phi$, where $\mathbf{i}: M_{1}^{n}(c) \rightarrow E_{t}^{n+1}$ is the canonical inclusion. Then

$$
\tau_{2}(\phi)=\tau_{2}(x)+2 c m \tau_{1}(x)+\left(2 m^{2}-c\left\langle\tau_{1}(x), \tau_{1}(x)\right\rangle\right) x .
$$

By Proposition 4.2, we have the following corollary (see [5, Corollary 4.2]).

Corollary 4.3. Let $\gamma: I \rightarrow M_{1}^{3}(c)$ be a unit speed curve. Then $\gamma$ is biharmonic if and only if $x(s)=\mathbf{i} \circ \gamma(s)$ satisfies

$$
x^{(i v)}+2 c\left\langle x^{\prime}, x^{\prime}\right\rangle x^{\prime \prime}+\left(1-c\left\langle\tau_{1}(\gamma), \tau_{1}(\gamma)\right\rangle\right) x=0 .
$$

By applying Corollary 4.3, we classify proper biharmonic curves in nonflat Lorentz space forms as follows. 
Theorem 4.4. Let $\gamma: I \rightarrow S_{1}^{3}(1)$ be a unit speed curve in de Sitter 3-space. Then $\gamma$ is proper biharmonic if and only if $x=\mathbf{i} \circ \gamma$ is congruent to one of the following five families.

(1) A spacelike curve with $\left\langle\tau_{1}(\gamma), \tau_{1}(\gamma)\right\rangle=0$;

$$
x(s)=\left(c_{1}+c_{2} s\right) \cos s+\left(c_{3}+c_{4} s\right) \sin s,
$$

where $c_{1}, c_{2}, c_{3}$ and $c_{4}$ are constant vectors orthogonal to each other satisfying $\left\langle c_{1}, c_{1}\right\rangle=\left\langle c_{3}, c_{3}\right\rangle=1$ and $\left\langle c_{2}, c_{2}\right\rangle=\left\langle c_{4}, c_{4}\right\rangle=0$; however, $\left\langle c_{2}, c_{2}\right\rangle^{2}+$ $\left\langle c_{4}, c_{4}\right\rangle^{2} \neq 0$.

(2) A spacelike circle with a spacelike principal normal vector field satisfying $\kappa=1$;

$$
x(s)=c_{1}+c_{2} \cos \sqrt{2} s+c_{3} \sin \sqrt{2} s,
$$

where $c_{1}, c_{2}$ and $c_{3}$ are constant vectors orthogonal to each other satisfying $\left\langle c_{1}, c_{1}\right\rangle=\left\langle c_{2}, c_{2}\right\rangle=\left\langle c_{3}, c_{3}\right\rangle=\frac{1}{2}$.

(3) A spacelike helix with a spacelike principal normal vector field satisfying $\kappa^{2}-\tau^{2}=1$;

$x(s)=c_{1} \cosh (\sqrt{\kappa-1} s)+c_{2} \sinh (\sqrt{k-1} s)+c_{3} \cos (\sqrt{\kappa+1} s)+c_{4} \sin (\sqrt{\kappa+1} s)$,

where $c_{1}, c_{2}, c_{3}$ and $c_{4}$ are constant vectors orthogonal to each other satisfying $\left\langle c_{1}, c_{1}\right\rangle=-\left\langle c_{2}, c_{2}\right\rangle=\left\langle c_{3}, c_{3}\right\rangle=\left\langle c_{4}, c_{4}\right\rangle=\frac{1}{2}$.

(4) A timelike circle satisfying $\kappa=1$;

$$
x(s)=c_{1}+c_{2} \cosh \sqrt{2} s+c_{3} \sinh \sqrt{2} s,
$$

where $c_{1}, c_{2}$ and $c_{3}$ are constant vectors orthogonal to each other satisfying $\left\langle c_{1}, c_{1}\right\rangle=\left\langle c_{2}, c_{2}\right\rangle=-\left\langle c_{3}, c_{3}\right\rangle=\frac{1}{2}$.

(5) A timelike helix satisfying $\kappa^{2}-\tau^{2}=1$;

$$
x(s)=c_{1} \cos \sqrt{\kappa-1} s+c_{2} \sin \sqrt{\kappa-1} s+c_{3} \cosh \sqrt{\kappa+1} s+c_{4} \sinh \sqrt{\kappa+1} s,
$$

where $c_{1}, c_{2}, c_{3}$ and $c_{4}$ are constant vectors orthogonal to each other satisfying $\left\langle c_{1}, c_{1}\right\rangle=\left\langle c_{2}, c_{2}\right\rangle=\left\langle c_{3}, c_{3}\right\rangle=-\left\langle c_{4}, c_{4}\right\rangle=\frac{1}{2}$.

Proof. We solve (4.1) under the condition that $\langle x, x\rangle=1,\left\langle x^{\prime}, x^{\prime}\right\rangle= \pm 1$ and $\tau_{1}(\gamma) \neq 0$, that is, $\left\langle x^{\prime}, x^{\prime}\right\rangle x^{\prime \prime}+x \neq 0$ for all $s$. We divide (4.1) into three types.

Case (i). $x$ is spacelike and $\left\langle\tau_{1}(\gamma), \tau_{1}(\gamma)\right\rangle=0$ : (4.1) is rewritten as

$$
x^{(i v)}+2 x^{\prime \prime}+x=0 \text {, }
$$

whose solutions are congruent to (1).

Case (ii). $x$ is a spacelike helix with a spacelike principal normal vector field satisfying $\kappa^{2}-\tau^{2}=1:(4.1)$ is rewritten as

$$
x^{(i v)}+2 x^{\prime \prime}+\left(1-\kappa^{2}\right) x=0 .
$$

If $\kappa=1$ (respectively, $\kappa>1$ ), then $x$ is congruent to (2) (respectively, (3)). 
Case (iii). $x$ is a timelike helix satisfying $\kappa^{2}-\tau^{2}=1:(4.1)$ is rewritten as

$$
x^{(i v)}-2 x^{\prime \prime}+\left(1-\kappa^{2}\right) x=0 .
$$

If $\kappa=1$ (respectively, $\kappa>1$ ), then $x$ is congruent to (4) (respectively, (5)).

THEOREM 4.5. Let $\gamma: I \rightarrow H_{1}^{3}(-1)$ be a unit speed curve in anti-de Sitter 3-space. Then $\gamma$ is proper biharmonic if and only if $x=\mathbf{i} \circ \gamma$ is congruent to one of the following five families.

(1) A spacelike curve with $\left\langle\tau_{1}(\gamma), \tau_{1}(\gamma)\right\rangle=0$;

$$
x(s)=\left(c_{1}+c_{2} s\right) e^{s}+\left(c_{3}+c_{4} s\right) e^{-s},
$$

where $c_{1}, c_{2}, c_{3}$ and $c_{4}$ are constant vectors satisfying $\left\langle c_{1}, c_{3}\right\rangle=-\frac{1}{2}$ and $\left\langle c_{i}, c_{j}\right\rangle=$ 0 unless $(i, j)=(1,3)$ or $(3,1)$; however, $\left\langle c_{2}, c_{2}\right\rangle^{2}+\left\langle c_{4}, c_{4}\right\rangle^{2} \neq 0$.

(2) A spacelike helix with a spacelike principal normal vector field satisfying $\kappa^{2}-\tau^{2}=-1$ and $\kappa \neq 0$;

$$
x(s)=e^{\alpha s}\left(c_{1} \cos \beta s+c_{2} \sin \beta s\right)+e^{-\alpha s}\left(c_{3} \cos \beta s+c_{4} \sin \beta s\right),
$$

where $\alpha$ and $\beta$ are constants satisfying $\alpha^{2}-\beta^{2}=1,2 \alpha \beta=\kappa$, and moreover $c_{1}, c_{2}, c_{3}$ and $c_{4}$ are constant vectors satisfying $\left\langle c_{1}, c_{3}\right\rangle=\left\langle c_{2}, c_{4}\right\rangle=-\frac{1}{2}$ and $\left\langle c_{i}, c_{j}\right\rangle=0$ unless $(i, j)=(1,3),(3,1),(2,4)$ or $(4,2)$.

(3) A spacelike circle with a timelike principal normal vector field satisfying $\kappa=1$;

$$
\gamma(s)=c_{1}+c_{2} \cosh \sqrt{2} s+c_{3} \sinh \sqrt{2} s,
$$

where $c_{1}, c_{2}$ and $c_{3}$ are constant vectors orthogonal to each other satisfying $\left\langle c_{1}, c_{1}\right\rangle=\left\langle c_{2}, c_{2}\right\rangle=-\left\langle c_{3}, c_{3}\right\rangle=-\frac{1}{2}$.

(4) A spacelike helix with a timelike principal normal vector field satisfying $\kappa^{2}+$ $\tau^{2}=1$ and $\kappa \neq 0$

$$
x(s)=c_{1} \cos \sqrt{1-\kappa} s+c_{2} \sin \sqrt{1-\kappa} s+c_{3} \cosh \sqrt{1+\kappa} s+c_{4} \sinh \sqrt{1+\kappa} s,
$$

where $c_{1}, c_{2}, c_{3}$ and $c_{4}$ are constant vectors orthogonal to each other satisfying $\left\langle c_{1}, c_{1}\right\rangle=\left\langle c_{2}, c_{2}\right\rangle=\left\langle c_{3}, c_{3}\right\rangle=-\left\langle c_{4}, c_{4}\right\rangle=-\frac{1}{2}$.

(5) A timelike helix satisfying $\kappa^{2}-\tau^{2}=-1$ and $\kappa \neq 0$;

$$
x(s)=e^{\alpha s}\left(c_{1} \cos \beta s+c_{2} \sin \beta s\right)+e^{-\alpha s}\left(c_{3} \cos \beta s+c_{4} \sin \beta s\right),
$$

where $\alpha$ and $\beta$ are constants satisfying $\alpha^{2}-\beta^{2}=-1,2 \alpha \beta=\kappa$, and moreover $c_{1}, c_{2}, c_{3}$ and $c_{4}$ are constant vectors satisfying $\left\langle c_{1}, c_{3}\right\rangle=\left\langle c_{2}, c_{4}\right\rangle=-\frac{1}{2}$ and $\left\langle c_{i}, c_{j}\right\rangle=0$ unless $(i, j)=(1,3),(3,1),(2,4)$ or $(4,2)$.

Proof. We solve (4.1) under the condition that $\langle x, x\rangle=1,\left\langle x^{\prime}, x^{\prime}\right\rangle= \pm 1$ and $\tau_{1}(\gamma) \neq 0$, that is, $\left\langle x^{\prime}, x^{\prime}\right\rangle x^{\prime \prime}-x \neq 0$ for all $s$. We divide (4.1) into four types. 
Case (i). $x$ is spacelike and $\left\langle\tau_{1}(\gamma), \tau_{1}(\gamma)\right\rangle=0$ : (4.1) is rewritten as

$$
x^{(i v)}-2 x^{\prime \prime}+x=0,
$$

whose solutions are congruent to (1).

Case (ii). $x$ is a spacelike helix with a spacelike principal normal vector field satisfying $\kappa^{2}-\tau^{2}=-1$ and $\kappa \neq 0:(4.1)$ is rewritten as

$$
x^{(i v)}-2 x^{\prime \prime}+\left(1+\kappa^{2}\right) x=0,
$$

whose solutions are congruent to (2).

Case (iii). $x$ is a spacelike helix with a timelike principal normal vector field satisfying $\kappa^{2}+\tau^{2}=1$ and $\kappa \neq 0:(4.1)$ is rewritten as

$$
x^{(i v)}-2 x^{\prime \prime}+\left(1-\kappa^{2}\right) x=0 .
$$

If $\kappa=1$ (respectively, $0<\kappa<1$ ), then $x$ is congruent to (3) (respectively, (4)).

Case (iv). $x$ is a timelike helix satisfying $\kappa^{2}-\tau^{2}=-1:(4.1)$ is rewritten as

$$
x^{(i v)}+2 x^{\prime \prime}+\left(1+\kappa^{2}\right) x=0,
$$

whose solutions are congruent to (5).

\section{Biharmonic surfaces in nonflat Lorentz 3-space forms}

By a similar computation to [4, Proof of Theorem 4.1] (see [6, Lemma 2.1]), we obtain the following lemma.

Lemma 5.1. Let $M^{2}$ be a pseudo-Riemannian surface in a Lorentz 3-space form of constant sectional curvature $c$. Then $M^{2}$ is biharmonic if and only if

$$
\left\{\begin{array}{l}
\Delta^{D} H=\left(2 c-\langle N, N\rangle \operatorname{trace} A_{N}^{2}\right) H, \\
2 \operatorname{trace} A_{D_{(\cdot)} H}(\cdot)+\operatorname{grad}(\langle H, H\rangle)=0,
\end{array}\right.
$$

where $N$ is a unit normal vector field.

We need the following two lemmas to classify proper biharmonic pseudoRiemannian surfaces in nonflat Lorentz 3-space forms.

Lemma 5.2. Let $M^{2}$ be a pseudo-Riemannian surface in a Lorentz 3-space form of constant sectional curvature c. Then $M^{2}$ is proper biharmonic if and only if it has nonzero constant mean curvature and $A_{N}$ satisfies

$$
2 c-\langle N, N\rangle \operatorname{trace} A_{N}^{2}=0 .
$$

Proof. Let $M^{2}$ be a proper biharmonic pseudo-Riemannian surface in $M_{1}^{3}(c)$ and let $\left\{e_{1}, e_{2}, e_{3}\right\}$ be a local orthonormal frame field such that $\left\{e_{1}, e_{2}\right\}$ are tangent to $M^{2}$ and $e_{3}$ is normal to $M^{2}$. We put $\epsilon_{i}=\left\langle e_{i}, e_{i}\right\rangle$ and $H=f e_{3}$. Note that $\epsilon_{1} \epsilon_{2} \epsilon_{3}=-1$. 
By Lemma 5.1,

$$
\begin{gathered}
\Delta_{M} f=\left(2 c-\epsilon_{3} \operatorname{trace} A^{2}\right) f, \\
A(\operatorname{grad} f)=-\epsilon_{3} f(\operatorname{grad} f),
\end{gathered}
$$

where $\Delta_{M} f=-\sum_{i=1}^{2}\left\langle e_{i}, e_{i}\right\rangle\left\{e_{i}\left(e_{i} f\right)-\left(\nabla_{e_{i}} e_{i}\right) f\right\}$ and $A=A_{e_{3}}$.

Let $U=\left\{p \in M^{2} \mid\left(\operatorname{grad} f^{2}\right)(p) \neq 0\right\}$. Assume that $U$ is nonempty. Since $-\epsilon_{3} f$ is an eigenvector of $A$ by (5.2) and $\operatorname{trace} A=2 \epsilon_{3} f$, the shape operator $A$ is diagonalisable and we can choose a local orthonormal frame field $\left\{e_{1}, e_{2}\right\}$ such that $e_{1}$ is parallel to $\operatorname{grad} f$. Then

$$
\begin{aligned}
& e_{2} f=0, \\
& h\left(e_{1}, e_{1}\right)=-\epsilon_{1} f e_{3}, \quad h\left(e_{1}, e_{2}\right)=0, \quad h\left(e_{2}, e_{2}\right)=3 \epsilon_{2} f e_{3}, \\
& \operatorname{trace} A^{2}=10 f^{2} .
\end{aligned}
$$

We put $\nabla e_{i}=\omega_{i}^{j} e_{j}$. Then $\omega_{1}^{2}=\omega_{2}^{1}$. From (5.3) and equation (2.3) of Codazzi,

$$
\omega_{1}^{2}\left(e_{1}\right)=0, \quad 3 e_{1} f=-4 f \omega_{1}^{2}\left(e_{2}\right) .
$$

By the first equation of (5.6), we can choose a local coordinate system $\{u, v\}$ such that $e_{1}=\partial / \partial u$ and $e_{2}$ is parallel to $\partial / \partial v$. Then, from (5.3), we have $f=f(u)$.

We denote by $f^{\prime}$ and $f^{\prime \prime}$ the first and the second derivatives of $f$ with respect to $x$. Using (5.6),

$$
4 f \Delta_{M} f=3 \epsilon_{1}\left(f^{\prime}\right)^{2}-4 \epsilon_{1} f f^{\prime \prime} .
$$

By combining (5.2), (5.5) and (5.7), we obtain

$$
4 f f^{\prime \prime}-3\left(f^{\prime}\right)^{2}+40 \epsilon_{2} f^{4}+8 c \epsilon_{1} f^{2}=0 .
$$

If we put $\theta=\left(f^{\prime}\right)^{2}$, then (5.8) can be rewritten as

$$
\frac{d \theta}{d f}-\frac{3}{2 f} \theta=-20 \epsilon_{2} f^{3}-4 c \epsilon_{1} f,
$$

which implies that

$$
\theta=-8 \epsilon_{2} f^{4}-8 \epsilon_{1} c f^{2}+C f^{\frac{3}{2}},
$$

where $C$ is a constant.

On the other hand, by (5.4), (5.6) and equation (2.2) of Gauss with $X=W=e_{1}$ and $Y=Z=e_{2}$,

$$
4 f f^{\prime \prime}-7\left(f^{\prime}\right)^{2}-16 \epsilon_{2} f^{4}-\frac{16}{3} c \epsilon_{1} f^{2}=0 .
$$

Combining (5.8) and (5.10) implies that

$$
\theta=-14 \epsilon_{2} f^{4}-\frac{10}{3} c \epsilon_{1} f^{2}
$$

By (5.9) and (5.11), $f$ is constant on $U$, which is a contradiction. Therefore, $U$ is empty, that is, $M^{2}$ has constant mean curvature. Hence, by (5.2), we get (5.1).

The converse is clear from Lemma 5.1. 
Lemma 5.3 [2]. Let $M^{2}$ be a surface with index 1 in $M_{1}^{3}(c)$ and let $(t-\lambda)^{2}, \lambda$ being a nonzero constant, be the minimal polynomial of its shape operator $A_{N}$. Then, in a neighbourhood of any point, $M^{2}$ is a B-scroll over a null curve given by

$$
x(s, u)=\gamma(s)+u Y(s),
$$

where $\gamma(s)$ is a null curve in $M_{1}^{3}(c) \subset E_{t}^{4}$ with an associated Cartan frame $\{X, Y, Z\}$. That is, $\{X, Y, Z\}$ is a pseudo-orthonormal frame field along $\gamma(s)$ :

$$
\begin{array}{ll}
\langle X, X\rangle=\langle Y, Y\rangle=0, & \langle X, Y\rangle=-1, \\
\langle X, Z\rangle=\langle Y, Z\rangle=0, & \langle Z, Z\rangle=1,
\end{array}
$$

such that

$$
\begin{aligned}
& \gamma^{\prime}(s)=X(s), \\
& Z^{\prime}(s)=-\lambda X(s)-k(s) Y(s),
\end{aligned}
$$

where $k(s) \neq 0$ for all $s$. Moreover, the shape operator $A_{N}$ of (5.12) is represented as

$$
A_{N}=\left(\begin{array}{cc}
\lambda & 0 \\
k(s) & \lambda
\end{array}\right)
$$

with respect to the usual frame $\{\partial / \partial s, \partial / \partial u\}$.

Contrary to Theorem 3.5, there exist proper biharmonic pseudo-Riemannian surfaces in nonflat Lorentz 3-space forms as follows.

THEOREM 5.4. Let $M^{2}$ be a pseudo-Riemannian surface in a Lorentz 3-space form of constant sectional curvature $c$, where $c \in\{-1,1\}$. Then $M^{2}$ is proper biharmonic if and only if it is congruent to one of the following:

(1) $S_{1}^{2}(2) \subset S_{1}^{3}(1)$;

(2) $H^{2}(-2) \subset H_{1}^{3}(-1)$;

(3) a B-scroll over a null curve of constant Gauss curvature 2 in $S_{1}^{3}(1)$.

Proof. Let $M^{2}$ be a proper biharmonic pseudo-Riemannian surface in $M_{1}^{3}(c)$, where $c \in\{-1,1\}$. By Lemma 5.2, the eigenvalues of the shape operator $A_{N}$ are constant. For simplicity, we put $A=A_{N}$.

Case (i). $A$ is diagonalisable: if $A$ has exactly two mutually distinct eigenvalues $\lambda$ and $\mu$, by [1, Theorem 3.5] we have that $c+\langle N, N\rangle \lambda \mu=0$. Combining this and (5.1) yields that $\lambda+\mu=0$, which is a contradiction with the assumption that $M^{2}$ is proper biharmonic. Therefore, $A=\lambda I$, where $I$ is the identity operator. Since $A$ satisfies (5.1), we obtain that $\lambda^{2}=1$. By virtue of [1, Theorem 5.1], $M^{2}$ is congruent to (1) or (2).

Case (ii). $A$ is not diagonalisable: in this case, the index of $M^{2}$ is equal to 1 and hence $\langle N, N\rangle=1$. If $A$ has a double real eigenvalue $\lambda$, then, by Lemma $5.3, M^{2}$ is congruent to a $B$-scroll over a null curve (5.12) with (5.13). It follows from (5.1) and (5.13) 
that $\lambda^{2}=c=1$. Therefore, $M^{2}$ is congruent to (3). If $A$ has complex eigenvalues $\alpha \pm \beta i$, then $A$ is represented as

$$
A=\left(\begin{array}{cc}
\alpha & -\beta \\
\beta & \alpha
\end{array}\right)
$$

with respect to an orthonormal frame. By equation (2.3) of Codazzi and (5.14), we find that $M^{2}$ is a flat surface. Hence, equation (2.2) of Gauss and (5.14) show that $c=-1$ and $\alpha^{2}+\beta^{2}=1$. In this case, the shape operator $A$ does not satisfy (5.1) because $M^{2}$ is nonharmonic.

The converse is verified by using Lemma 5.1.

\section{References}

[1] N. Abe, N. Koike and S. Yamaguchi, 'Congruence theorems for proper semi-Riemannian hypersurfaces in a real space form', Yokohama Math. J. 35 (1987), 123-136.

[2] L. J. Alias, A. Ferrandez and P. Lucas, '2-type surfaces in $S_{1}^{3}$ and $H_{1}^{3}$ ', Tokyo J. Math. 17 (1994), 447-454.

[3] A. Balmuş, 'Biharmonic Maps and Submanifolds', in: Balkan Society of Geometers, Differential Geometry - Dynamical Systems, Monographs (Geometry Balkan Press, Bucharest, 2009).

[4] R. Caddeo, S. Montaldo and C. Oniciuc, 'Biharmonic submanifolds of $S^{3}$, Internat. J. Math. 12 (2001), 867-876.

[5] R. Caddeo, S. Montaldo and C. Oniciuc, 'Biharmonic submanifolds in spheres', Israel J. Math. 130 (2002), 109-123.

[6] B.-Y. Chen and S. Ishikawa, 'Biharmonic surfaces in pseudo-Euclidean spaces', Mem. Fac. Sci. Kyushu Univ. Ser. A Math. 45 (1991), 325-349.

[7] B.-Y. Chen and S. Ishikawa, 'Biharmonic pseudo-Riemannian submanifolds in pseudo-Euclidean spaces', Kyushu J. Math. 52 (1998), 167-185.

[8] J. Eells and J. H. Sampson, 'Harmonic mappings of Riemannian manifolds', Amer. J. Math. 86 (1964), 109-160.

[9] J. Inoguchi, 'Biharmonic curves in Minkowski 3-space', Internat. J. Math. Math. Sci. 21 (2003), $1365-1368$.

[10] G. Y. Jiang, '2-harmonic maps and their first and second variational formulas', Chinese Ann. Math. Ser. A 7 (1986), 389-402, English translation, Note Mat. 28(Suppl. No. 1) (2008), 209-232.

[11] S. Montaldo and C. Oniciuc, 'A short survey on biharmonic maps between Riemannian manifolds', Rev. Un. Mat. Argentina 47(2) (2006), 1-22, available at http://inmabb.criba.edu.ar/revuma/.

[12] T. Sasahara, 'Quasi-minimal Lagrangian surfaces whose mean curvature vectors are eigenvectors', Demonstratio Math. 38 (2005), 185-196.

[13] T. Sasahara, 'Biharmonic Lagrangian surfaces of constant mean curvature in complex space forms', Glasg. Math. J. 49 (2007), 487-507.

\section{TORU SASAHARA, General Education and Research Center, Hachinohe Institute of Technology, Hachinohe, Aomori 031-8501, Japan e-mail: sasahara@hi-tech.ac.jp}

